\title{
Asymptotic expansions and domain decomposition
}

\author{
G. Geymonat ${ }^{1}$, S. Hendili ${ }^{2},{ }^{3}$, F. Krasucki ${ }^{3}$, M. Serpilli ${ }^{4}$ and M. Vidrascu ${ }^{2}$
}

\section{Introduction}

At a first glance asymptotic expansions and domain decomposition are two alternatives to efficiently solve multi scale elasticity problems. In this paper we will combine these two methods: we will use, for several types of problems, asymptotic expansions and show that for an efficient implementation of problems obtained at the asymptotic limit it may be useful to use domain decomposition type algorithms. In particular we will consider problems with heterogenous or non heterogenous thin layers(see Fig 1 a) et b)). To directly solve such problems by a standard finite element method is too expensive from a computational point of view. That is why specific asymptotic expansions are used and allow to replace the original problem by a set of problems defined on a new domain where the thin layer is replaced by a line in 2D or a surface in 3D (see Fig $1 \mathrm{c}$ )). In addition particular jumping conditions are defined on this new interface yielding a non standard problem which can be solved by a Neumann-Neumann domain decomposition algorithm. The paper is organized as follows: In Section 2 we review of a domain decomposition algorithm on an elasticity problem, in Section 3 we consider a thin layer of heterogeneities which can be holes or elastic inclusions and, finally, in Section 4 we consider a multi-materials with a thin layer with high ratio in material properties.

\section{Domain decomposition algorithm: general setting for an elasticity problem}

The aim of this paragraph is to specify the notations. We consider a standard linear elasticity problem:

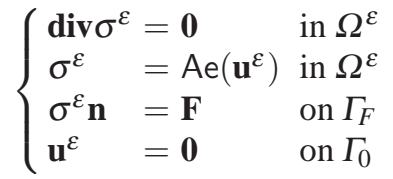

LMS, UMR-CNRS 7649, Ecole Polytechnique giuseppe. geymonat @ lms . polytechnique.fr . EPI REO, INRIA Rocquencourt, marina.vidrasculinria.fr, soufiane.hendili@inria.fr . I3M, UMR-CNRS 5149, Université Montpellier 2 krasucki@math.univ-montp2.fr. Departement of Civil and Building construction engineering, and architecture, Università Politecnica delle Marche, Ancona m.serpilli@univpm. it 
The mechanical characteristics of the multi-material structure are described by the elasticity tensor A. Each material is isotropic but A is indeed material dependent. In the sequel we will omit this constitutive equation. The structure is clamped on a part $\Gamma_{0} \subset \partial \Omega$ (of surface measure $>0$ ) and a density $\mathbf{F}$ of surface forces is applied on the complementary part $\Gamma_{F}$. In a variational form this problem writes

$$
A(\mathbf{u}, \mathbf{v})=L(\mathbf{v}) \text { for all } \mathbf{v} \in V, \text { with } A(\mathbf{u}, \mathbf{v})=\int_{\Omega} A^{i j k \ell} e_{k \ell}(\mathbf{u}) e_{i j}(\mathbf{v}) d x .
$$

Let us mention that the variational form is always used to discretize the problem, nevertheless in order to simplify notations we will use either partial differential equations or variational form. The same problem will be considered in sections 3 and 4, where the the domain differs with respect to the heterogeneities. We will explain how the domain decomposition algorithm is adapted in each situation. In order to use a primal domain decomposition to solve the problem we transform the problem on the entire domain in a problem on the interface. After splitting the domain in non overlapping subdomains we introduce an additional unknown, $\lambda=\operatorname{Tr}(\mathbf{u})$ on the interface. For simplicity reasons we will consider here only two sub-domains and only a first level preconditioner. To solve the original problem is equivalent to solving the following problem on each subdomain:

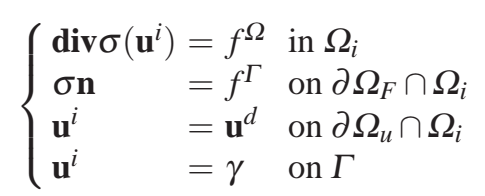

By linearity $\mathbf{u}^{i}=\mathbf{u}_{0}^{i}+\mathbf{u}_{\gamma}^{i}$ where $\mathbf{u}_{0}^{i}$ is the solution of (3) with $\mathbf{u}_{0}^{i}=0$ on $\Gamma$ and $\mathbf{u}_{\gamma}^{i}$ is the solution of (3) with $f^{\Omega}=0, f^{\Gamma}=0$. In order to settle the interface problem we write the continuity of the normal stress on the interface:

$$
\sigma\left(\mathbf{u}^{1}\right) \mathbf{n}^{1}+\sigma\left(\mathbf{u}^{2}\right) \mathbf{n}^{2}=\sigma\left(\mathbf{u}_{\gamma}^{1}\right) \mathbf{n}^{1}+\sigma\left(\mathbf{u}_{0}^{1}\right) \mathbf{n}^{1}+\sigma\left(\mathbf{u}_{\gamma}^{2}\right) \mathbf{n}^{2}+\sigma\left(\mathbf{u}_{0}^{2}\right) \mathbf{n}^{2}=0
$$

Using the Steklov Poincaré operator $S$ which is defined as follows: for $\gamma$ given on $\Gamma$ (the sub-domains interface )

$$
S_{i} \gamma=\sigma\left(\mathbf{u}_{\gamma}^{i}\right) \mathbf{n}^{i}
$$

where $\mathbf{n}^{i}$ denotes the outer normal on $\Gamma$, the interface problem writes:

$$
S_{1} \gamma+S_{2} \gamma=-\sigma\left(\mathbf{u}_{0}^{1}\right) \mathbf{n}^{1}-\sigma\left(\mathbf{u}_{0}^{2}\right) \mathbf{n}^{2}
$$

In variational form

$$
S_{1}(\gamma, \mathbf{v})+S_{2}(\gamma, \mathbf{v})=-L\left(\sigma\left(\mathbf{u}_{0}^{1}\right) \mathbf{n}^{1}, \mathbf{v}\right)-L\left(\sigma\left(\mathbf{u}_{0}^{2}\right) \mathbf{n}^{2}, \mathbf{v}\right)
$$

This problem will be solved using a iterative method, the preconditioner is $M=$ $\alpha_{1} S_{1}^{-1}+\alpha_{2} S_{2}^{-1}$ with $\alpha_{1}+\alpha_{2}=1$. ([6], [3]) 
The parallel between this approach and the one used in the asymptotic analysis (as described in 1) is that a particular problem has to be solved on the interface, the next sections will specify this concept.

\section{Structure with a thin layer of heterogeneities}

Let us consider a three-dimensional structure with small identical heterogeneities periodically distributed along a surface $\omega$. Let $\varepsilon$ be a small dimensionless parameter which characterizes the diameter and the periodic arrangement of the heterogeneities. We denote $B^{\varepsilon}$ the layer of thickness $\varepsilon$ containing the heterogeneities centered on $\omega$ (see Fig. 1 a)).

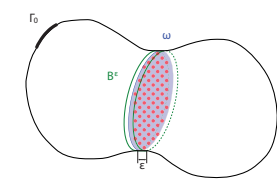

Fig. 1 a) Heterogeneous layer

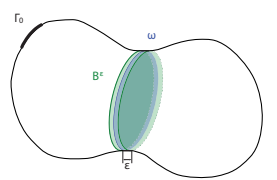

b) Homogeneous layer

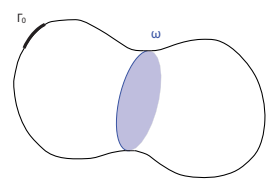

c) Limit domain

The domain $\Omega$ contains $I^{\varepsilon}$ the set of identical heterogeneities of diameter $\varepsilon D$ and $\varepsilon$-periodically distributed in the vicinity of the interior surface $\omega$ of equation $x_{1}=0$. We consider the problem (3) with two types of inclusions: cavities and elastic inclusions. The displacement field $\mathbf{u}^{\varepsilon}$ and the stress field $\sigma^{\varepsilon}$, satisfy, respectively, equilibrium equation (1).

Notice that $\Omega$ is a domain with a number of heterogeneities which depends on $\varepsilon$. For the elastic inclusions $A^{S}$ and $A^{I}$ (the elasticity tensor in the structure, respectively in the inclusions) are of same order of magnitude.

The asymptotic analysis of this problem for $\varepsilon \rightarrow 0$ provides a model describing the linear elastic behavior of the structure on a simplified domain denoted by $\Omega_{0}$ where the layer $B^{\varepsilon}$ becomes the surface $\Gamma$ (see Fig. 1 c)). More precisely, by assuming that $\mathbf{u}^{\varepsilon} \simeq \mathbf{u}^{0}+\varepsilon \mathbf{u}^{1}$, the initial problem (1) is approximated by two new ones where the layer of heterogeneities is replaced by a surface on which particular jump conditions are defined.

The zeroth order approximation $\mathbf{u}^{0}$ is the solution of the following transmission linear problem :

$$
\left\{\begin{array}{lll}
\operatorname{div} \sigma^{0}=\mathbf{0} & \text { in } \Omega_{0} \\
\sigma^{0} \mathbf{n} & =\mathbf{F} & \text { on } \Gamma_{F} \\
\mathbf{u}^{0} & =\mathbf{0} & \text { on } \Gamma
\end{array}\right.
$$

Notice that there are no jumps on $\Gamma$ for the outer approximation. In other words, at the zero order the outer approximation does not consider the heterogeneities. Thus this problem can be solved using a standard finite element procedure. 
The first order approximation $\mathbf{u}^{1}$ is the unique solution of the following boundary value problem (with transmissions conditions on $\Gamma$ ):

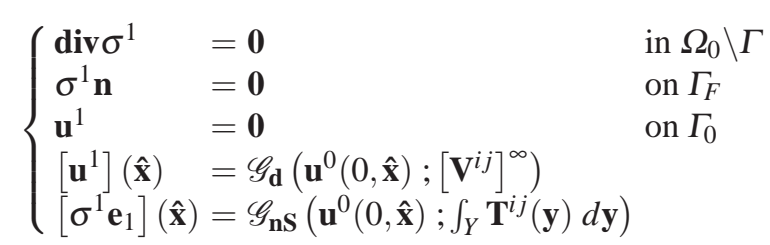

where $\mathbf{V}^{i j}$ are the solutions of nine elementary problems defined on one representative cell $Y([5],[4])$ and $\mathbf{T}^{i j}$ are the stress fields associated with $\mathbf{V}^{i j}$ and $\left[\mathbf{V}^{i j}\right]^{\infty}=\lim _{y_{1} \rightarrow+\infty} \mathbf{V}^{i j}-\lim _{y_{1} \rightarrow-\infty} \mathbf{V}^{i j-}$

$\mathscr{G}_{\mathbf{d}}$ has the same structure for the different types of inclusions, while $\mathscr{G}_{\mathbf{n S}}$ depends on the inclusion:

$$
\mathscr{G}_{\mathbf{d}}=\frac{\partial u_{i}^{0}}{\partial x_{j}}(0, \hat{\mathbf{x}})\left[\mathbf{V}^{i j}\right]^{\infty}
$$

i) in the elastic inclusions case one has:

$$
\mathscr{G}_{\mathbf{n S}}=\operatorname{div}\left(|I|\left(\mathrm{A}^{S}-\mathrm{A}^{I}\right) \mathrm{e}\left(\mathbf{u}^{0}(0, \hat{\mathbf{x}})\right)-\frac{\partial u_{i}^{0}}{\partial x_{j}}(0, \hat{\mathbf{x}}) \int_{Y} \mathbf{T}^{i j}(\mathbf{y}) d \mathbf{y}\right)
$$

ii) in the cavities case one has:

$$
\mathscr{G}_{\mathbf{n S}}=\operatorname{div}\left(|I| \mathrm{A}^{I} \mathrm{e}\left(\mathbf{u}^{0}(0, \hat{\mathbf{x}})\right)-\frac{\partial u_{i}^{0}}{\partial x_{j}}(0, \hat{\mathbf{x}}) \int_{Y} \mathbf{T}^{i j}(\mathbf{y}) d \mathbf{y}\right)
$$

Let us emphasize that, for the first order problem, $\mathscr{G}_{\mathbf{d}}$ and $\mathscr{G}_{\mathbf{n S}}$ are given and depend on the first and second order derivatives of the zeroth order problem. This is not an issue at the domain decomposition level, while, at the implementation level, since the solution $\mathbf{u}^{\mathbf{0}}$ is only of class $C^{0}$, a regularization is needed. In practice, an efficient way to implement the jump conditions in problem (6) is to solve this problem by a domain decomposition type algorithm which will be detailed hereafter.

Finally, the generic form of the first order problem, (6) is given by:

$$
\left\{\begin{array}{llll}
-\operatorname{div} \sigma(\mathbf{u}) & =\mathbf{0} & & \text { in } \Omega \\
\sigma \mathbf{n} & =0 & & \text { on } \partial \Omega_{F} \\
\mathbf{u} & =0 & & \text { on } \partial \Omega_{u} \\
{[\mathbf{u}]} & =\mathscr{G}_{\mathbf{d}} & & \text { on } \Gamma \\
{[\sigma \mathbf{n}]} & =\mathscr{G}_{\mathbf{n S}} & & \text { on } \Gamma
\end{array}\right.
$$

where $\mathscr{G}_{\mathbf{d}}$ and $\mathscr{G}_{\mathbf{n S}}$ denote, respectively, the gap in displacements and normal stresses on $\Gamma$. By using the linearity of the problem, we will search, in each subdomain a solution of the form

$$
\mathbf{u}^{i}=\mathbf{w}^{i}+\beta_{i} \mathbf{z}^{i}
$$

where $\beta_{i}$ are two real numbers conveniently chosen and $\mathbf{z}^{i}$ are the solutions of the following two independent problems: 


$$
\left\{\begin{array}{lll}
-\operatorname{div} \sigma\left(\mathbf{z}^{i}\right)=0 & \text { in } \Omega_{i} \\
\sigma \mathbf{n} & =0 & \text { on } \partial \Omega_{F} \cap \Omega_{i} \\
\mathbf{z}^{i} & =0 & \text { on } \partial \Omega_{u} \cap \Omega_{i} \\
\mathbf{z}^{i} & =\mathscr{G}_{\mathbf{d}} & \text { on } \Gamma
\end{array}\right.
$$

Notice that

$$
-\operatorname{div} \sigma\left(\mathbf{w}^{\mathbf{i}}\right)=-\operatorname{div} \sigma\left(\mathbf{u}^{i}-\beta_{i} \mathbf{z}^{i}\right)=0
$$

The transmission conditions for $\mathbf{w}^{i}$ are given by:

$$
\begin{cases}{[\mathbf{w}]=[\mathbf{u}]-\beta_{1} \mathscr{G}_{\mathbf{d}}+\beta_{2} \mathscr{G}_{\mathbf{d}}} & =\left(1-\beta_{1}+\beta_{2}\right) \mathscr{G}_{\mathbf{d}} \\ {[\sigma \mathbf{n}]=[\mathbf{u}]+\beta_{1} \sigma\left(\mathbf{z}^{1}\right) \mathbf{n}-\beta_{2} \sigma\left(\mathbf{z}^{2}\right) \mathbf{n}=\mathscr{G}_{\mathbf{n S}}+\beta_{1} \sigma\left(\mathbf{z}^{1}\right) \mathbf{n}-\beta_{2} \sigma\left(\mathbf{z}^{2}\right) \mathbf{n}}\end{cases}
$$

If we choose $1-\beta_{1}+\beta_{2}=0$ then $\mathbf{w}$ is continuous on the interface $\Gamma$, while the normal stress is discontinuous at the interface.

By introducing the Steklov Poincaré, as described above, the unknown $\gamma$ on the interface is the solution of the following problem:

$$
\left(S_{1}+S_{2}\right) \gamma=-\sigma\left(\mathbf{w}_{0}^{1}\right) \mathbf{n}^{1}-\sigma\left(\mathbf{w}_{0}^{2}\right) \mathbf{n}^{2}+\mathscr{G}_{\mathbf{n S}}+\beta_{1} \sigma\left(\mathbf{z}^{1}\right) \mathbf{n}^{1}-\beta_{2} \sigma\left(\mathbf{z}^{2}\right) \mathbf{n}^{2}
$$

Let us remark that this equation differs from (4) only on the right hand side. In this situation the solution of the entire problem is not as regular as in section 3 . Here, because of the jumps, the solution is not in $H^{1}(\Omega)$, this is why the norms used in the following numerical simulations are $L^{2}(\Omega)$. Thus as the operator does not change, the same algorithms (and in particular the same preconditioner) may be used to solve the problem with the same performance and no additional analysis is required to prove efficiency.
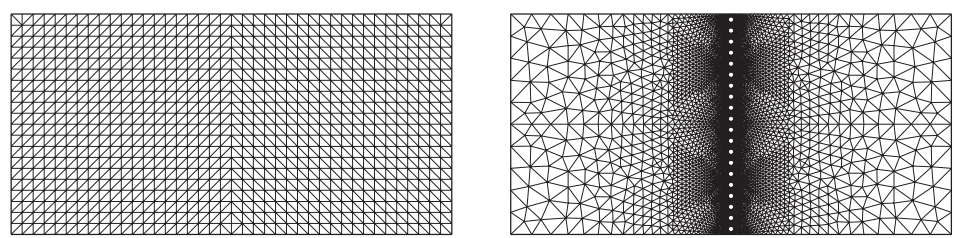

Fig. 2 a) Mesh used for the asymptotic computation b) Fine mesh for $\varepsilon=\frac{1}{20}$

In order to numerically validate this approach we consider a $2 \mathrm{D}$ case where $\Omega$ is a plane domain containing $\mathscr{N}^{\varepsilon}$ holes of diameter $\varepsilon D$ Notice that the domain and thus the number of holes depends on $\varepsilon$. A reference solution $\mathbf{u}_{h}^{\varepsilon}$ of the problem (1) is computed on a large mesh (see Fig 2 b)) and compared with the asymptotic solution $\mathbf{u}_{h}^{0}$ and $\mathbf{u}_{h}^{0}+\varepsilon \mathbf{u}_{h}^{1}$ obtained by solving the problems (5) and (6) on a corse mesh (see Fig. 2 a)). This comparison is performed by computing the relative error for the $L^{2}$-norm (see table (1)). 
Table $1 L^{2}$-errors norms computed in $\Omega^{\varepsilon}$

\begin{tabular}{|c|c|c|c|c|}
\hline$\varepsilon$ & $\mathrm{Nb}$ elements & dofs & $\frac{\left\|\mathbf{u}_{h}^{\varepsilon}-\mathbf{u}_{h}^{0}\right\|_{L^{2}}}{\left\|\mathbf{u}_{h}^{\varepsilon}\right\|_{L^{2}}}$ & $\frac{\left\|\mathbf{u}_{h}^{\varepsilon}-\left(\mathbf{u}_{h}^{0}+\varepsilon \mathbf{u}_{h}^{1}\right)\right\|_{L^{2}}}{\left\|\mathbf{u}_{h}^{\varepsilon}\right\|_{L^{2}}}$ \\
\hline $1 / 20$ & 13348 & 54938 & 0.013501216 & 0.001225971 \\
\hline $1 / 40$ & 27668 & 113530 & 0.006689361 & 0.000475813 \\
\hline $1 / 80$ & 57164 & 234050 & 0.003281498 & 0.000176916 \\
\hline
\end{tabular}

\section{Multimaterials with strong curved interface}

In this section we analyze the mechanical behavior of a particular structural assembly, which is constituted by an elastic shell-like inclusion with high rigidity surrounded by two three-dimensional elastic bodies.

Let $\Omega^{+}$and $\Omega^{-}$be two disjoint open domains with smooth boundaries $\partial \Omega^{+}$and $\partial \Omega^{-}$. Let $\omega:=\left\{\partial \Omega^{+} \cap \partial \Omega^{-}\right\}^{\circ}$ be the interior of the common part of the boundaries which is assumed to be a non empty domain in $\mathbb{R}^{2}$. Let $\theta \in \mathscr{C}^{2}\left(\bar{\omega} ; \mathbb{R}^{3}\right)$ be an immersion such that the vectors $\mathbf{a}_{\alpha}(y):=\partial_{\alpha} \theta(y)$ form the covariant basis of the tangent plane to the surface $S:=\theta(\bar{\omega})$. We note with $\mathbf{a}_{3}(y):=\frac{\mathbf{a}_{1}(y) \wedge \mathbf{a}_{2}(y)}{\left|\mathbf{a}_{1}(y) \wedge \mathbf{a}_{2}(y)\right|}$ the unit normal vector to $S$. We insert an intermediate curved layer moving $\Omega^{+}$and $\Omega^{-}$in the $\mathbf{a}_{3}$ and $-\mathbf{a}_{3}$ directions, respectively, by an amount equal to $t^{\varepsilon}>0$, where $\varepsilon$ is a small dimensionless real parameter. Then let $\Omega^{ \pm, \varepsilon}:=\left\{x^{\varepsilon}:=x \pm t^{\varepsilon} \mathbf{a}_{3} ; x \in \Omega^{ \pm}\right\}$, $\left.\Omega^{m, \varepsilon}:=\omega \times\right]-t^{\varepsilon}, t^{\varepsilon}$, and $\Omega^{\varepsilon}:=\Omega^{-, \varepsilon} \cup \Omega^{+, \varepsilon} \cup \Omega^{m, \varepsilon}$, as shown in Fig. 1 The structure is clamped on $\Gamma_{0}^{\varepsilon} \subset\left(\partial \Omega^{\varepsilon} \backslash \Gamma^{m, \varepsilon}\right)$. We consider that $S$ coincides with the middle surface of the shell-like inclusion $\Omega^{m, \varepsilon}$. Moreover, the shell thickness $t^{\varepsilon}$ depends linearly on $\varepsilon$, so that $t^{\varepsilon}=\varepsilon t$. For a more detailed treatment of this asymptotic problem in a general curvilinear framework, the reader can refer to [1], [2].

The physical variational problem defined over the variable domain $\Omega^{\varepsilon}$ is

$$
\left\{\begin{array}{l}
\text { Find } \mathbf{u}^{\varepsilon} \in V^{\varepsilon}:=\left\{\mathbf{v}^{\varepsilon} \in H^{1}\left(\Omega^{\varepsilon} ; \mathbb{R}^{3}\right) ; \mathbf{v}^{\varepsilon} \mid \Gamma_{0}^{\varepsilon}=\mathbf{0}\right\} \\
A_{-}^{\varepsilon}\left(\mathbf{u}^{\varepsilon}, \mathbf{v}^{\varepsilon}\right)+A_{+}^{\varepsilon}\left(\mathbf{u}^{\varepsilon}, \mathbf{v}^{\varepsilon}\right)+A_{m}^{\varepsilon}\left(\mathbf{u}^{\varepsilon}, \mathbf{v}^{\varepsilon}\right)=L\left(\mathbf{v}^{\varepsilon}\right) \text { for all } \mathbf{v}^{\varepsilon} \in V^{\varepsilon},
\end{array}\right.
$$

where $\mathrm{A}$ is defined as in (2).

The functional $L(\cdot)$ is the linear form associated with the applied forces. Here $A^{i j k \ell, \varepsilon}:=\lambda^{\varepsilon} g^{i j, \varepsilon} g^{k \ell, \varepsilon}+\mu^{\varepsilon}\left(g^{i k, \varepsilon} g^{j \ell, \varepsilon}+g^{i \ell, \varepsilon} g^{j k, \varepsilon}\right)$ are the contravariant components of the elasticity tensor, where $g^{i j}$ can be considered as the curvilinear version of the Kronecker's delta. Let us suppose that the Lamé's constants of the isotropic materials satisfy the following dependences with respect to $\varepsilon: \lambda^{ \pm, \varepsilon}=\lambda^{ \pm}, \mu^{ \pm, \varepsilon}=$ $\mu^{ \pm}, \lambda^{m, \varepsilon}=\frac{1}{\varepsilon} \lambda^{m}, \mu^{m, \varepsilon}=\frac{1}{\varepsilon} \mu^{m}$.

As shown in [1], the asymptotic expansion method applied to the physical problem (12) leads to a simplified model for the assembly, in which the layer inclusion is reduced to its middle surface as $\varepsilon$ tends to zero. Thus the presence of the layer is replaced by a surface shell like energy at the interface which corresponds to a partic- 
ular membrane transmission condition between the two three-dimensional bodies The main result is contained in the following theorem:

Theorem 1. The leading term $\mathbf{u}^{0}$ of the asymptotic expansion $\mathbf{u}(\varepsilon)=\mathbf{u}^{0}+\varepsilon \mathbf{u}^{1}+$ $\varepsilon^{2} \mathbf{u}^{2}+\ldots$, is the unique solution of the following limit problem:

$$
\left\{\begin{array}{l}
\text { Find } \mathbf{u}^{0} \in V_{M} \text { such that } \\
A^{-}\left(\mathbf{u}^{0}, \mathbf{v}\right)+A^{+}\left(\mathbf{u}^{0}, \mathbf{v}\right)+A_{M}^{m}\left(\mathbf{u}^{0}, \mathbf{v}\right)=L(\mathbf{v}) \text { for all } \mathbf{v} \in V_{M}
\end{array}\right.
$$

where $V_{M}:=\left\{\mathbf{v} \in H^{1}\left(\Omega^{+} \cup \omega \cup \Omega^{-} ; \mathbb{R}^{3}\right) ;\left.\mathbf{v}\right|_{\omega} \in H^{1}\left(\omega ; \mathbb{R}^{2}\right) \times H^{\frac{1}{2}}(\omega),\left.\mathbf{v}\right|_{\Gamma_{0}}=\mathbf{0}\right\}$, and

$$
A_{M}^{m}\left(\mathbf{u}^{0}, \mathbf{v}\right)=2 t \int_{\omega} a^{\alpha \beta \sigma \tau} e_{\sigma \tau}\left(\mathbf{u}^{0}\right) e_{\alpha \beta}(\mathbf{v}) d y
$$

is the bilinear form associated with the membrane behavior of the shell, $a^{\alpha \beta \sigma \tau}$ is the elasticity tensor of the shell and $e_{\alpha \beta}(\mathbf{u}):=\frac{1}{2}\left(u_{\beta \mid \alpha}+u_{\alpha \mid \beta}\right)$ is the change of metric tensor.

Remark. In the simplified model we obtain a membrane transmission condition at the interface between the two three-dimensional bodies, which can be interpreted as a curvilinear generalization of the Ventcel-type transmission condition obtained in [1]. Indeed, by integrating by parts problem (13), one has

$$
\left\{\begin{array} { l l } 
{ - \operatorname { d i v } \sigma _ { \pm } = \mathbf { f } } & { \text { in } \Omega ^ { \pm } , } \\
{ \mathbf { u } ^ { 0 } = \mathbf { 0 } } & { \text { on } \Gamma _ { 0 } , }
\end{array} \quad \left\{\begin{array}{l}
{\left[\sigma^{\alpha 3}\right]=\operatorname{div}\left(n^{\alpha \beta}\right) \text { in } \omega,} \\
{\left[\sigma^{33}\right]=n^{\alpha \beta} b_{\alpha \beta} \text { in } \omega,}
\end{array}\right.\right.
$$

where $\sigma_{ \pm}^{i j}:=A_{ \pm}^{i j k \ell} e_{k \ell}\left(\mathbf{u}^{0}\right)$ and $n^{\alpha \beta}:=2 t a^{\alpha \beta \sigma \tau} e_{\sigma \tau}\left(\left.\mathbf{u}^{0}\right|_{\omega}\right)$ represent, respectively, the Cauchy stress tensor and the membrane stress tensor of the shell, $\left[\sigma^{i 3}\right]:=\sigma_{+}^{i 3}-\sigma_{-}^{i 3}$ represents the stress jump at the interface $\omega$, and $b_{\alpha \beta}$ is the second fundamental form associated to the shell middle surface.

In order to solve the problem (13) we introduce a specific domain decomposition algorithm, more precisely, we construct the interface problem. We consider three subdomains $\Omega^{+}:=\Omega^{(1)}, \Omega^{-}:=\Omega^{(2)}$, and the shell $\Omega^{m}$. For the two 3D domains, $\Omega^{1}, \Omega^{2}$ we introduce the corresponding Steklov Poincaré operator and we observe that the domain $\Omega^{3}$ is the interface. Thus, in a variational form, the compatibility condition on the interface writes :

$$
S^{1}(\gamma, \mathbf{v})+S^{2}(\gamma, \mathbf{v})+A_{M}^{m}(\gamma, \mathbf{v})=L\left(-\sigma\left(\mathbf{u}_{0}^{1}\right) \mathbf{n}^{1}-L \sigma\left(\mathbf{u}_{0}^{2}\right) \mathbf{n}^{2}, \mathbf{v}\right)
$$

This problem can be solved by a Neumann-Neumann algorithm as well because, compared to (4) we add in the right hand side a term wich is symmetric and positive defined.

As a numerical example, we consider an axisymmetric problem of two thick cylinders bonded together with a cylindrical shell with high rigidity subjected to an internal pressure $\left(E_{c y l}=5 e 05, E_{\text {shell }}=5 e 07, v=0.3, \mathrm{t}=0.1, R_{\text {max }}=6\right)$. We choose this particular geometry because it is characterized by an immediate mechanical interpretation. Moreover we can compute an exact solution for this problem. We tested the domain decomposition by using two subdomains (the shell is "glued" 
to another subdomain) and three subdomains and by studying the influence of a Neumann-Neumann preconditioner on the number of iterations. The preliminary results are shown in the following table. As we can see the number of iterations decreases drastically when adopting a preconditioner.

Table 2 Mesh: $N_{e l}=11020, N_{e l, \text { shell }}=580$

\begin{tabular}{lll}
\hline Subdomains & Iterations & Iterations with preconditioner \\
\hline 2 & 69 & 6 \\
$2+1($ shell $)$ & 70 & 46 \\
\hline
\end{tabular}

In the actual simulations we can use membrane or shell elements. The shell is more robust but also more computationally demanding. In our example we used a membrane element. The drawback is that the operator is not invertible (that is needed in the preconditioning step) and that explains why the results with two domains are far better than with three domains. Hence, our test example does not behave totally as a pure membrane. This feature disappears when shell elements are used or when the problem has a pure membrane behavior.

Acknowledgements This work was partially supported by the French Agence Nationale de la Recherche (ANR) under Grant Epsilon (BLAN08-2 312370) (Domain decomposition and multiscale computations of singularities in mechanical structures).

\section{References}

1. Bessoud, A.L., Krasucki, F., Serpilli, M.: Asymptotic analysis of shell-like inclusions with high rigidity. J. Elasticity 103(2), 153-172 (2011). DOI 10.1007/s10659-010-9278-1. URL http://dx.doi.org/10.1007/s10659-010-9278-1

2. Chapelle, D., Ferent, A.: Modeling of the inclusion of a reinforcing sheet within a 3D medium. Math. Models Methods Appl. Sci. 13(4), 573-595 (2003). DOI 10.1142/S0218202503002635. URL http://dx.doi.org/10.1142/S0218202503002635

3. De Roeck, Y.H., Le Tallec, P., Vidrascu, M.: A domain-decomposed solver for nonlinear elasticity. Comput. Methods Appl. Mech. Engrg. 99(2-3), 187-207 (1992). DOI 10.1016/ 0045-7825(92)90040-Q. URL http://dx.doi.org/10.1016/0045-7825 (92) 90040-Q

4. Geymonat, G., Hendili, S., Krasucki, F., Vidrascu, M.: The matched asymptotic expansion for the computation of the effective behavior of an elastic structure with a thin layer of holes. International Journal for Multiscale Computational Engineering 9(5), 529-542 (2011). DOI 10. 1615/IntJMultCompEng.v9.i5. URL http://hal.inria.fr/inria-00540992/en

5. Geymonat, G., Hendili, S., Krasucki, F., Vidrascu, M.: Matched asymptotic expansion method for an homogenized interface model. (2012). URL http: // hal archives-ouvertes. fr/hal-00757005. Submitted

6. Le Tallec, P.: Domain decomposition methods in computational mechanics. Comput. Mech. Adv. 1(2), 121-220 (1994) 